Sādhanā Vol. 39, Part 4, August 2014, pp. 765-783. (C) Indian Academy of Sciences

\title{
AI-based adaptive control and design of autopilot system for nonlinear UAV
}

\author{
ANIL KUMAR YADAV* and PRERNA GAUR \\ Division of Instrumentation and Control Engineering, Netaji Subhas Institute of \\ Technology, New Delhi, 110 078, India \\ e-mail: anilei007@gmail.com; prernagaur@yahoo.com
}

MS received 2 September 2013; revised 21 March 2014; accepted 19 May 2014

\begin{abstract}
The objective of this paper is to design an autopilot system for unmanned aerial vehicle (UAV) to control the speed and altitude using electronic throttle control system (ETCS) and elevator, respectively. A DC servo motor is used for designing of ETCS to control the throttle position for appropriate amount of air mass flow. Artificial Intelligence (AI)-based controllers such as fuzzy logic PD, fuzzy logic PD + I, self-tuning fuzzy logic PID (STF-PID) controller and fuzzy logic-based sliding mode adaptive controller (FLSMAC) are designed for stable autopilot system and are compared with conventional PI controller. The target of throttle, speed and altitude controls are to achieve a wide range of air speed, improved energy efficiency and fuel economy with reduced pollutant emission. The energy efficiency using specific energy rate per velocity of UAV is also presented in this paper.
\end{abstract}

Keywords. Autopilot system; AI-based control; DC servo motor; electronic throttle control system; sliding mode control and UAV.

\section{Introduction}

One of the most difficult aspects of manually controlled aerial vehicle is the coupling between the control over the vehicle speed and altitude. These output variables cannot be controlled independently to each other through the aerial vehicle control devices like elevator and throttle (Amelink et al 2003). The proper coordination between output variables and control devices are obtained using autopilot system. An unmanned aerial vehicle (UAV) commonly known as a drone is an aircraft or aerial vehicle without a human pilot on board. UAV flights are either controlled autonomously by computers in the vehicle, or under the remote control, or pilot on the ground or in another vehicle. In recent years, due to successful implementation of UAVs may be observed in military and the wide range of applications such as monitoring of traffic, crops and pollutions, crime and border surveillance; reconnaissance, coast guard, fisheries and environment protection, security of national assets, transport and communications, etc. UAVs significantly

*For correspondence 
reduce the cost and risk to human life mainly in nuclear or chemical leakage inspection and are preferred in dull, dirty or dangerous for manned aerial vehicle missions (Manathara \& Debasish 2011; Kurnaz et al 2009; Chao et al 2010; Orsag et al 2011; Gu et al 2006; Bateman et al 2011; Shiau \& Ma 2009).

The stability in terms of energy of an UAV with automatic throttle and elevator controls has been investigated in this paper, where it is required to control height or altitude by means of the elevator, and speed using electronic throttle control system (ETCS). The controlling of throttle position using electric motor drives is called ETCS (Yadav \& Gaur 2014). The UAV with the ETCS can give the subsonic, transonic, supersonic and hypersonic speeds (Stevens \& Lewis 2003) and the dependent variables on throttle position like pollution, fuel consumption, energy efficiency are also improved (Yadav et al 2011a). The throttle is used as a valve to regulate air inflow into the engine combustion system and it determines the power developed by the engine by controlling the amount of air mass entering the engine cylinders. An UAV engine that produces thrust operates over a wide range of speed and the fuel/air mixture can be adjusted for maximum efficiency (Vasak et al 2007). The aerial vehicle with ETCS and nonlinear dynamics make the complete system as nonlinear and unstable. The linear control techniques cannot be applied to control this problem due to high nonlinearities in the system. In literature, many advanced control techniques such as fuzzy logic control (FLC) (Kurnaz et al 2009), adaptive control (Liu et al 2008; Han et al 2012; Osborne et al 2011), Artificial neural networks (Kamalasadan \& Ghandakly 2011), robust control Wang \& Stengel (2000, 2005), disturbance observer-based control (Liu et al 2013), nonlinear energy-based control (Akmeliawati \& Mareels 2010), self-organizing control (Shin \& Xu 2009), sliding mode control, $\mathrm{H}_{\infty}$ control and some other control techniques have been used for autonomous control of UAVs or similar system like aircraft to guarantee desirable trajectory (Keviczky \& Balas 2006; Ducard 2009; Hull 2009; Yadav \& Gaur 2013; Goerzen et al 2010; Kontogiannis \& Ekaterinaris 2013). During military, transport and launching of satellites assignment the UAV is often unloaded at one or more points, each time part of the UAV is unloaded there is seldom a measuring device to obtain new accurate mass measurements. The various disturbances parameters such as wind speed, air density, mass, altitude and centre of gravity position, etc. are present during the flight of UAV. The mass or weight is generally several magnitudes larger than the other parameters. So for this research, the common approach is adopted, that all the parametric uncertainties can be aggregated as an uncertainty in mass and variation of air density. However, UAVs are normally operated in a dynamic environment of different air density, weather and load conditions which create the uncertainties and disturbances. The design of control algorithms must be robust and adaptive with respect to uncertainties so that the controller should be capable of handling the parameter uncertainties.

The Artificial Intelligence (AI)-based controller such as fuzzy logic control (Goerzen et al 2010; Yadav et al 2011b), self-tuning fuzzy logic PID controller (Yadav \& Gaur 2014; Zulfatman \& Rahmat 2009) which are designed to tune the value of $K_{P}, K_{i}$ and $K_{d}$ of the PID controller and fuzzy logic based sliding mode adaptive controller (FLSMAC) are designed and implemented for UAV in this paper. FLSMAC combines the merits of sliding-mode control, the fuzzy inference mechanism and the adaptive algorithm. First a PID, PD or integral type slidingmode switching surface is designed and then a fuzzy sliding-mode controller is investigated in which a simple fuzzy inference mechanism is used to determine the equivalent control law. The various applications of FLSMAC such as manipulators, aircraft, servo drives, hybrid electric vehicles and robot are presented in (Li \& Xu 2010; Sun et al 2011; Zhan \& Zhou 2013; Wai et al 2004; Subudhi \& Ge 2012; Asif et al 2014). The time domain performance parameters such as overshoot (OS), settling time (ST) and rise time (RT) are used as a measure of autopilot system 
of UAV performance. The integral error performance indices (IEPI) such as integral of absolute error (IAE) and integral of square of error (ISE) are also studied to get better all-round performance for autopilot system of UAV. For the better performance analysis of a system, it is required to have proper balance in time domain and IEPI. All the above mentioned performance specifications are considered in this paper. Comparative performance analysis of controllers for UAV is presented in order to identify the superior controller over other controllers designed in this paper. A mathematical software 'MATLAB' is used for comparative performance analysis.

\section{Problem formulation and mathematical modelling}

The motion of a UAV in free flight is extremely complicated. To reduce the complexity of analysis, we usually assume that the UAV is a rigid-body and the motion of UAV consists of small deviation from its equilibrium (trimmed) flight condition. In addition, we assume that the UAV equations of motion can be separated into two groups, namely, longitudinal equations and lateral equations. In this paper, we have considered the longitudinal dynamics only. Using the standard notation of Stevens \& Lewis (2003), the equations of motion are written with respect to the body axis of the UAV (Stevens \& Lewis 2003; Ducard 2009; Hull 2009). The nomenclature used in this paper is described in table 1.

The nonlinear UAV dynamics, consisting of force, moment, and kinematic equations, can be compactly written as: Force equations:

$$
\dot{u}=r v-q w+\frac{1}{m} F_{x}, \dot{v}=-r u+p w+\frac{1}{m} F_{y} \text { and } \dot{w}=-p v+q u+\frac{1}{m} F_{z} .
$$

Table 1. Nomenclature.

\begin{tabular}{|c|c|c|c|c|c|}
\hline Symbol & Description & SI units & Symbol & Description & SI units \\
\hline$u, v, w$ & Velocity in the $\mathrm{x}, \mathrm{y}, \mathrm{z}$ body axis & $\mathrm{m} / \mathrm{s}$ & $R_{a}$ & Armature resistance & $\Omega$ \\
\hline$F_{x}, F_{y}, F_{z}$ & Force in the $\mathrm{x}, \mathrm{y}, \mathrm{z}$ body axis & $\mathrm{N}$ & $L_{a}$ & Armature inductance & $\mathrm{H}$ \\
\hline$p, q, r$ & Roll, Pitch, Yaw rate & $\mathrm{rad} . / \mathrm{s}$ & $K_{b}$ & Back EMF constant & Vs/rad. \\
\hline$\phi, \theta, \psi$ & Roll, Pitch, Yaw angle & rad. & $K_{t}$ & Motor torque constant & $\mathrm{Nm} / \mathrm{A}$ \\
\hline$\alpha, \beta$ & Angle of attack, sideslip angle & rad. & $K_{s p}$ & Throttle spring constant & $\mathrm{Nm} / \mathrm{rad}$. \\
\hline$\gamma$ & flight path angle & rad & $R_{a f}$ & Focal point of airflow on plate & $\mathrm{m}$ \\
\hline$\delta_{e}, \delta_{T}$ & Elevator, Throttle deflections & rad. & $\delta_{T_{0}}$ & Pre-tension angle of spring & rad. \\
\hline$L, M, N$ & Roll, pitch, yaw moment & $\mathrm{kg} \cdot \mathrm{m}^{2}$ & $R_{p}$ & Throttle plate radius & $\mathrm{m}$ \\
\hline$I_{x x}, I_{y y}, I_{z z}$ & Inertia about $\mathrm{x}, \mathrm{y}, \mathrm{z}$ axis & $\mathrm{kg} \cdot \mathrm{m}^{2}$ & $B_{m}$ & Motor damping constant & $\mathrm{Nms} / \mathrm{rad}$ \\
\hline$C_{L}, C_{M}$ & Lift, pitching moment coeff. & - & $B_{t}$ & Throttle damping constant & $\mathrm{Nms} / \mathrm{rad}$ \\
\hline$S, S_{\text {prop }}$ & Wing area, Propeller area & $\mathrm{m}^{2}$ & $J_{m}$ & Motor inertia & $\mathrm{kg} \cdot \mathrm{m}^{2}$ \\
\hline$c$ & Mean chord & $\mathrm{m}$ & $J_{g}$ & Throttle inertia & $\mathrm{kg} \cdot \mathrm{m}^{2}$ \\
\hline$m$ & Mass of UAV & $\mathrm{kg}$ & $J$ & Equivalent inertia & $\mathrm{kg} \cdot \mathrm{m}^{2}$ \\
\hline$\rho$ & Air density & $\mathrm{kg} / \mathrm{m}^{3}$ & V & Total velocity or speed of UAV & $\mathrm{m} / \mathrm{s}$ \\
\hline$*$ & Desired or reference value & - & $h$ & Altitude & $\mathrm{m}$ \\
\hline
\end{tabular}


Moment equations:

$$
\begin{aligned}
\dot{p} & =\frac{1}{I_{x x}}\left[L-\left(I_{z z}-I_{y y}\right) q r+I_{x z}(\dot{r}+p q)\right], \\
\dot{q} & =\frac{1}{I_{y y}}\left[M-\left(I_{x x}-I_{z z}\right) p r+I_{x z}\left(p^{2}-r^{2}\right)\right] \text { and } \\
\dot{r} & =\frac{1}{I_{z z}}\left[N-\left(I_{y y}-I_{x x}\right) p q+I_{x z} \dot{p}-I_{x z} q r .\right.
\end{aligned}
$$

Kinematic equation:

$$
\begin{aligned}
\dot{\theta} & =q \cos \phi-r \sin \phi, \quad \dot{\phi}=p+q \sin \phi \tan \theta+r \cos \phi \tan \theta \text { and } \\
\dot{\Psi} & =r \cos \phi / \cos \theta+q \sin \phi / \cos \theta .
\end{aligned}
$$

The definition of the body axis velocities are

$$
u=V \cos \alpha \cos \beta, \quad v=V \sin \beta \quad \text { and } \quad w=V \sin \alpha \cos \beta .
$$

The total velocity or airspeed $V$, angle of attack $\alpha$, and angle of sideslip $\beta$ can be expressed in term of body axis velocities as

$$
\begin{gathered}
V=|V|=\left(u^{2}+v^{2}+w^{2}\right)^{1 / 2}, \\
\alpha=\tan ^{-1} \frac{w}{u} \quad \text { and } \quad \beta=\sin ^{-1} \frac{v}{V} .
\end{gathered}
$$

The longitudinal motion of the UAV is considered in this paper. The output states and control inputs are $[h, V, \theta, \alpha, q]$ and $\left[\delta_{e}, \delta_{T}\right]$, respectively. The trimming conditions for longitudinal motion are assumed as $\beta=0, \theta=\alpha, \varphi=0, p=q=r=0$. For longitudinal motion, the force balance along the $\mathrm{x}$-direction of the body axis is as shown in figure 1, and represented in the form of equation as:

$$
F_{x}=F_{\text {Weight }}+F_{\text {Lift }}+F_{\text {Thrust }}+F_{\text {Drag }} .
$$

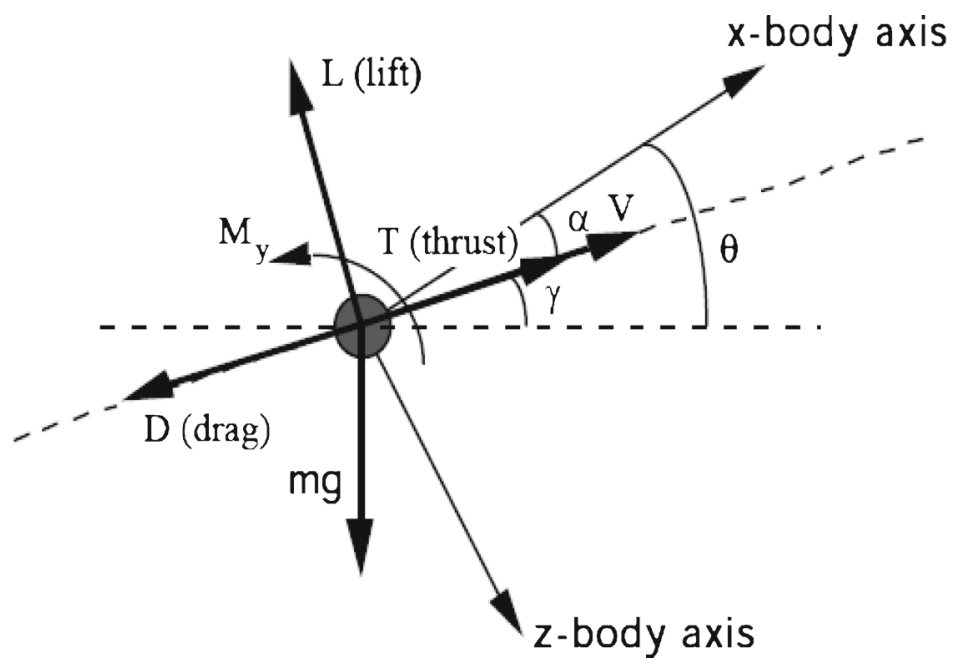

Figure 1. Forces on UAV in longitudinal motion. 
Writing the force equation in expended form (Manathara \& Debasish 2011):

$F_{x}=-m g \sin \theta+\frac{1}{2} \rho V^{2} S\left(C_{L_{0}}+C_{L_{\alpha}} \alpha+C_{L_{q}} \frac{c q}{2 V}+C_{L_{\delta_{e}}} \delta_{e}\right)+\frac{1}{2} \rho S_{\text {prop }} k_{\text {Engine }}^{2} C_{X_{\delta_{T}}}^{2} \delta_{T}^{2}-\frac{1}{2} \rho S_{\text {prop }} V^{2}$,

where $k_{\text {Engine }}$ and $C_{X_{\delta_{T}}}$ are dimensionless engine parameters, $C_{L_{0}}, C_{L_{\alpha}}, C_{L_{q}}$ and $C_{L_{\delta_{e}}}$ are lift control derivative of the UAV model. Here $m$ is total mass of aerial vehicle and defined as $m_{U A V}+m_{\text {fuel }}$, where $m_{\text {fuel }}$ decreases linearly at the rate of $1.1 \mathrm{~kg} / \mathrm{km}$. After applying trim conditions the simplified form of (1) is as

$$
V=\left[\frac{2 m g \sin \alpha-\rho S_{\text {prop }} k_{\text {Engine }}^{2} C_{X_{\delta_{T}}}^{2} \delta_{T}^{2}}{\rho S\left(C_{L_{0}}+C_{L_{\alpha}} \alpha+C_{L_{\delta_{e}}} \delta_{e}\right)-\rho S_{\text {prop }}}\right]^{0.5}
$$

The differential equation for the pitch angle $\theta$ and altitude $h$ are as follows:

$$
\begin{gathered}
\dot{\theta}=q \\
\dot{h}=V \sin \gamma .
\end{gathered}
$$

The flight path angle is denoted by $\gamma=\tan ^{-1}(w / u)=\tan ^{-1}(V \sin \alpha / V \cos \alpha)$, where $V=$ $\sqrt{u^{2}+w^{2}}$ and $\alpha=(\theta-\gamma)$ (Akmeliawati \& Mareels 2010; Hull 2009).

The pitching moment $M$ is written as (Wang \& Stengel 2000; Hull 2009)

$$
M=\frac{1}{2} \rho V^{2} S c\left(C_{M_{0}}+C_{M_{\alpha}} \alpha+C_{M_{q}} \frac{c q}{2 V}+C_{M_{\delta_{e}}} \delta_{e}\right),
$$

where $C_{M_{0}}, C_{M_{\alpha}}, C_{M_{q}}$ and $C_{M_{\delta_{e}}}$ are pitching moment control derivative of the UAV model. After applying trim condition on (12), yield (13)

$$
\alpha=-\left(C_{M_{0}}+C_{M_{\delta_{e}}} \delta_{e}\right) / C_{M_{\alpha}} .
$$

Equations (9) to (13) give the complete model of nonlinear UAV. The Mechatronic system of UAV engine that provides thrust force, with electronic throttle control system (ETCS) is shown in figure 2.

In figure 2 the throttle position is shown with $\delta_{T}$ and if $\delta_{T}$ is controlled the kinetic energy given by $E_{k}=(1 / 2) m V^{2}$ may be controlled using (9). In figure 2 the ETCS consists of DC servo motor to rotate the throttle plate. Here the DC servo motor is controlled by the applied motor voltage $E_{a}$ (Yadav \& Gaur 2014; Vasak et al 2007).

$$
\frac{d i_{a}}{d t}=\left(\frac{1}{L_{a}}\right)\left(-R_{a} i_{a}-K_{b} \frac{d \theta_{m}}{d t}+E_{a}\right),
$$

where $i_{a}$ is armature current (A), $\theta_{m}$ is armature angular position (rad). The back EMF $E_{b}$ due to the motor rotation is $K_{b}\left(d \theta_{m} / d t\right)$. The meaning of symbols used in equations is given in table 1 . The motor and rotational dynamics of throttle is as follows:

$$
\begin{gathered}
\frac{d^{2} \theta_{m}}{d t^{2}}=\left(\frac{1}{J_{m}}\right)\left(-B_{m}\left(\frac{d \theta_{m}}{d t}\right)-T_{L}+T_{m}\right), \\
\frac{d^{2} \delta_{T}}{d t^{2}}=\left(\frac{1}{J_{g}}\right)\left(-T_{s p}-B_{t}\left(\frac{d \delta_{T}}{d t}\right)-T_{a}+T_{g}\right),
\end{gathered}
$$




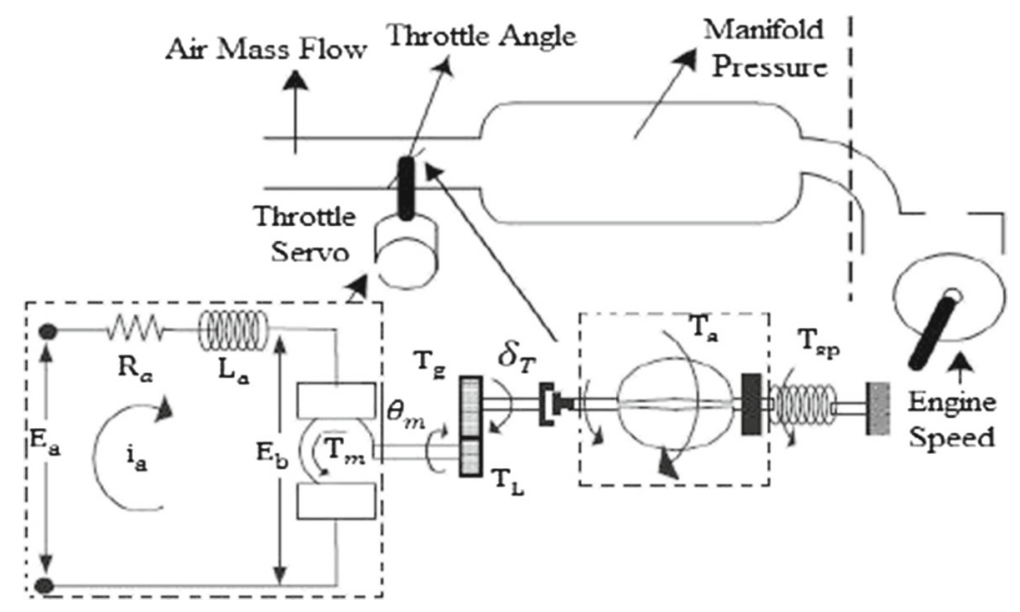

Figure 2. Mechatronic system of UAV engine with ETCS.

where $N$ is the gear ratio which governs the engine speed that is defined as $N=\theta_{m} / \delta_{T}=T_{g} / T_{L}$. $T_{a}$ is torque due to airflow $(\mathrm{Nm}), T_{g}$ is the torque transmitted from gears $(\mathrm{Nm}), T_{L}$ is the load torque $(\mathrm{Nm}), T_{m}$ is the torque applied by motor $(\mathrm{Nm}), T_{s p}$ is the return spring torque $(\mathrm{Nm})$ and $\delta_{T}$ is the throttle plate angular position in radians. The motor torque $T_{m}$ is defined as

$$
T_{m}=K_{t} i_{a}
$$

From (14) to (17)

$$
\begin{gathered}
\frac{d i_{a}}{d t}=\left(\frac{1}{L_{a}}\right)\left(-R_{a} i_{a}-K_{b} N \frac{d \delta_{T}}{d t}+e_{a}\right), \\
\frac{d^{2} \delta_{T}}{d t^{2}}=\left(\frac{1}{J}\right)\left(-B\left(\frac{d \delta_{T}}{d t}\right)+N T_{m}-T_{s p}-T_{a}\right),
\end{gathered}
$$

where $J=N^{2} J_{m}+J_{g}$ and $B=N^{2} B_{m}+B_{t}$. A stiff rotational spring returns the plate to a closed position when $E_{a}$ is zero. The spring assembly has been initialized to an angle $\delta_{T_{0}}$ which produces a closing torque. The spring torque $T_{s p}$ may be described as

$$
T_{s p}=K_{s p}\left(\delta_{T}+\delta_{T_{0}}\right) .
$$

The airflow over the throttle plate induces a small torque $T_{a}$ such as

$$
T_{a}=R_{a f} F_{a} \cos \delta_{T}
$$

The variable $F_{a}$ denotes the air force acting on the plate parallel to the air flow direction which is given by (22)

$$
F_{a}=\Delta P A_{p} \cos \delta_{T}
$$

where $\Delta P=P_{a t m}-P_{m}$, throttle plate area $\left(\mathrm{m}^{2}\right) \quad A_{p}=\pi R_{p}^{2}$ and $P_{m}=f\left(\delta_{T}, P_{a t m}, N\right)$. The manifold pressure $P_{m}$ is a nonlinear throttle angle dependent function that approaches to atmospheric pressure $\left(P_{\text {atm }}\right)$ as the throttle approaches a wide-open state. Hence $(21)$ is given as

$$
T_{a}=R_{a f} \Delta P \pi R_{p}^{2} \cos ^{2} \delta_{T}
$$




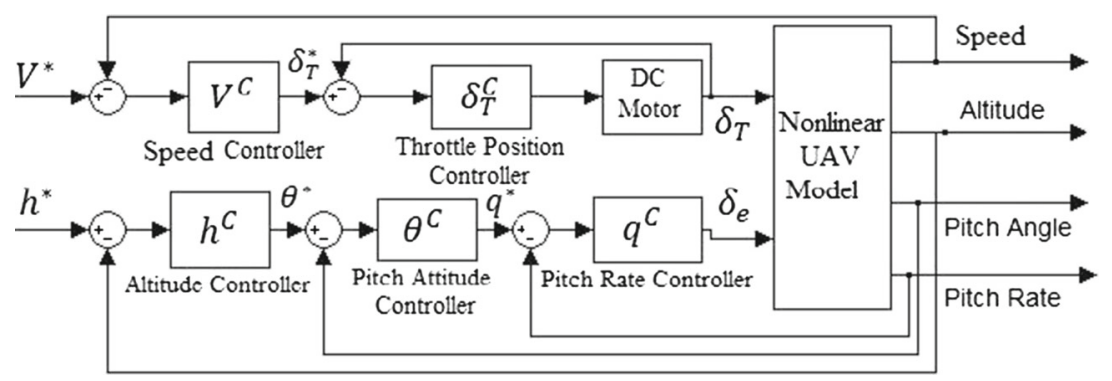

Figure 3. Autopilot system for longitudinal motion of UAV.

Equations (17) to (23) present complete model of nonlinear ETCS. The complete block diagram of autopilot system for longitudinal motion of UAV is represented by figure 3. It contains a DC motor that is a part of ETCS. The nonlinear UAV model was augmented with an innerloop controller based on pitch rate, pitch angle and throttle position feedback. The inner-loop controller has to stabilize the pitch and throttle valve. The simulation values in SI unit of UAV and ETCS are listed in table 2.

The value of air density $\rho$ as given in table 2 is a mean value and it is considered as unpredictable parameter and varying randomly. The Mach number ' $M_{a}$ ' is defined as the quotient of aerial vehicle speed $V$ and local speed of sound ' $a$ ' and given as $M_{a}=V / a$ (Wang \& Stengel 2005). The $M_{a}$ related with air speeds such as $M_{a}<1,0.8<M_{a} \leq 1.2,1<M_{a}<5$ and $5 \leq M_{a}$ for subsonic, transonic, supersonic and hypersonic speeds, respectively.

\section{Controller design and implementation}

In this section, conventional PI, Fuzzy logic PD (FL-PD), Fuzzy logic PD+I, self-tuning fuzzy PID (STF-PID) controllers and fuzzy logic based sliding mode adaptive controller (FLSMAC) design algorithm are presented which are used in autopilot design for UAV. PI controllers are widely used in industrial control applications which have a simple structure and can offer a satisfactory performance over a wide range of operation. The conventional tuning of PI controller like Ziegler Nichols does not apply for all nonlinear, time varying systems, hence the PID controller is tuned by hand tuning rule with the help of fuzzy logic. This may be called self-tuning fuzzy logic PID controller. The transfer function of generalized PID controller is given in (Yadav \& Gaur 2014; Yadav et al 2011a) and is written below:

$$
C(s)=K_{P}+\frac{K_{i}}{s}+K_{d} s=K_{P}\left(1+\frac{1}{T_{i} s}+T_{d} s\right)
$$

where $K_{p}, K_{i}$, and $K_{d}$ are proportional, integral and derivative gains, respectively. $T_{i}$ is reset time which is equal to $K_{p} / K_{i}$, and $T_{d}$ is rate time which is equal to $K_{d} / K_{P}$.

Table 2. Numerical values of UAV used in simulation.

\begin{tabular}{lccccccccccccc}
\hline$C_{L_{0}}$ & $C_{L_{\alpha}}$ & $C_{L_{q}}$ & $C_{L_{\delta_{e}}}$ & $C_{X_{\delta_{T}}}$ & $C_{M_{0}}$ & $C_{M_{\alpha}}$ & $C_{M_{\delta_{e}}}$ & $\rho$ & $k_{\text {Engine }}$ & $S_{\text {prop }}$ & $S$ & $m$ & $c$ \\
0.28 & -2.7 & 0.0 & 0.83 & 1 & 0.15 & -0.17 & -0.88 & 1.27 & 20 & 4.5 & 36 & 5000 & 1.98 \\
$R_{a}$ & $L_{a}$ & $K_{b}$ & $N$ & $K_{t}$ & $K_{s p}$ & $R_{a f}$ & $R_{p}$ & $B_{m}$ & $B_{t}$ & $J_{m}$ & $J_{g}$ & $J$ & $B$ \\
2 & 0.003 & 0.11 & 4 & 0.1 & 0.4 & 0.002 & 0.0015 & 0.03 & 0.002 & 0.001 & 0.005 & 0.021 & 0.482 \\
\hline
\end{tabular}




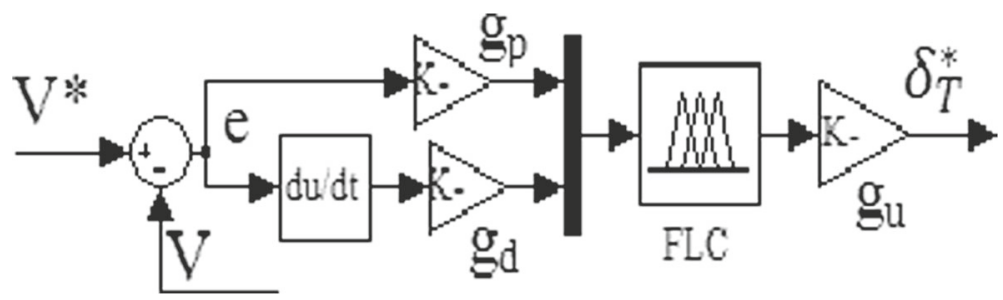

(a)

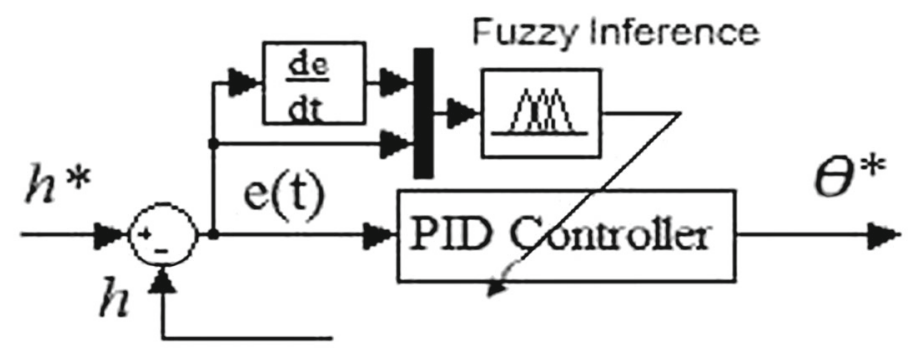

(b)

Figure 4. (a) Structure of FL-PD controller. (b) Structure of self-tuning fuzzy PID controller.

The FLC can be designed with knowledge base of the input and output variables of the controller. For designing the FLC four components are used namely rule base, inference engine, fuzzification and defuzzification (Yadav \& Gaur 2013; Yadav et al 2011b). The proposed structure of FL-PD controller is given in figure 4(a). The inputs to the FLC are the error $e(t)=$ $V^{*}(t)-V(t)$ and change in error that is $\dot{e}(t)$ and output variable is the desired throttle position $\delta_{T}^{*}$. The universe of discourse of the variables cover a range of $[-2,2]$ for error, $[-4,4]$ for change in error and $[-10,10]$ for output. A standard choice for the triangular membership functions is used with three membership functions for the three fuzzy variables (meaning $9=3^{2}$ rules in the rule base) as three membership functions are found sufficient to design the controller in this case. The resulting rule base is shown in the table 3. To obtain the crisp output, the center of gravity defuzzification method is used.

\subsection{Self-tuning fuzzy PID controller}

In STF-PID controller, the three parameters of PID controller $K_{p}, K_{i}$ and $K_{d}$ are tuned by using fuzzy logic (Yadav \& Gaur 2014; Zulfatman \& Rahmat 2009), because often the gains of the PID controller are not properly tuned for the desired UAV altitude with unpredictable parameter variations like density of air and mass. The conventional PID training rules are not effective when there is high altitude uncertainty. Hence, it is necessary to tune the PID controller parameters using STF-PID. The structure of the proposed self-tuning fuzzy PID controller is shown

Table 3. Fuzzy rule bases for FL-PD.

\begin{tabular}{lccc}
\hline$e \backslash \dot{e}$ & $\mathrm{P}$ & $\mathrm{ZE}$ & $\mathrm{N}$ \\
\hline $\mathrm{P}$ & $\mathrm{PB}$ & $\mathrm{P}$ & $\mathrm{ZE}$ \\
$\mathrm{ZE}$ & $\mathrm{P}$ & $\mathrm{ZE}$ & $\mathrm{N}$ \\
$\mathrm{N}$ & $\mathrm{ZE}$ & $\mathrm{N}$ & $\mathrm{NB}$ \\
\hline
\end{tabular}


in figure 4b. For designing and implementation of self-tuning fuzzy logic PID controller, there are two inputs to fuzzy inference that are error in altitude $e_{h}(t)=h^{*}(t)-h(t)$ and derivative of error in altitude $\dot{e}_{h}(t)$, and three outputs for each PID controller parameter $K_{p}^{\prime}$, $K_{i}^{\prime}$ and $K_{d}^{\prime}$ respecttively. In this controller, the designing process of the rules is based on properties of the PID controller and the characteristic of the plant (Yadav \& Gaur 2014).

Assume that the range of PID controller parameters $K_{p}, K_{i}, K_{d}$ are $\left[K_{p \min }, K_{\text {pmax }}\right]$, [ $K_{\text {imin }}$, $\left.K_{\text {imax }}\right]$, [ $\left.K_{d \min }, K_{d \max }\right]$, respectively. The range of each parameter is considered from the knowledge base and hence the range of PID controller parameters $K_{p}, K_{i}$ and $K_{d}$ is taken between 0.01 and 0.1 . These parameters are calibrated as follows: (Yadav \& Gaur 2014; Zulfatman \& Rahmat 2009)

$$
\begin{aligned}
& K_{p}^{\prime}=\frac{K_{p}-K_{p \min }}{K_{p \max }-K_{p \min }}=\frac{K_{p}-0.01}{0.1-0.01}, \quad K_{i}^{\prime}=\frac{K_{i}-K_{i \min }}{K_{i \max }-K_{i \min }}=\frac{K_{i}-0.01}{0.1-0.01}, \quad \text { and } \\
& K_{d}^{\prime}=\frac{K_{d}-K_{d \min }}{K_{d \max }-K_{d \min }}=\frac{K_{d}-0.01}{0.1-0.01} .
\end{aligned}
$$

After simplification of (25), we get new PID controller parameters which are:

$$
K_{p}=0.99 K_{p}^{\prime}+0.01, \quad K_{i}=0.99 K_{i}^{\prime}+0.01, \text { and } K_{d}=0.99 K_{d}^{\prime}+0.01 .
$$

The universe of discourse for input is between -0.1 and 0.1 , for output variables it is 0 to 1 and the triangular membership functions for inputs and outputs are selected. The fuzzy rules for STF-PID are shown in table 4.

3.2 Fuzzy logic based sliding mode adaptive controller (Yadav \& Gaur 2014; Li \& Xu 2010; Sun et al 2011; Zhan \& Zhou 2013; Wai et al 2004; Subudhi \& Ge 2012; Asif et al 2014)

Sliding mode control (SMC) is a variable structure control with high frequency discontinuous control action that switches between several functions depending on the system states (Yadav \& Gaur 2014). It is one of the most effective and robust nonlinear control techniques for autopilot system of UAV. The principle of SMC is to define a control law to drive the nonlinear state trajectory onto a switching surface and to maintain this trajectory sliding on this surface for all subsequent time (Yadav \& Gaur 2014). The control law is based on fuzzy logic and Lyapunov theory to guarantee the motion of the state trajectory towards the sliding surface. This is done by choosing a hitting control gain to maintain the derivative of Lyapunov function which is always a negative definite.

In order to design FLSMAC, first an integral type sliding surface is designed and defined as follows:

$$
s(t)=e(t)+\lambda \int e(t) d t,
$$

Table 4. Rule base matrix for STF-PID.

\begin{tabular}{llllll}
\hline$e_{h} \backslash \dot{e}_{h}$ & $\mathrm{NB}$ & $\mathrm{NS}$ & $\mathrm{ZE}$ & $\mathrm{PS}$ & $\mathrm{PB}$ \\
\hline $\mathrm{NB}$ & $\mathrm{S}$ & $\mathrm{S}$ & $\mathrm{MS}$ & $\mathrm{MS}$ & $\mathrm{M}$ \\
$\mathrm{NS}$ & $\mathrm{S}$ & $\mathrm{MS}$ & $\mathrm{MS}$ & $\mathrm{M}$ & $\mathrm{MB}$ \\
$\mathrm{ZE}$ & $\mathrm{MS}$ & $\mathrm{MS}$ & $\mathrm{M}$ & $\mathrm{MB}$ & $\mathrm{MB}$ \\
$\mathrm{PS}$ & $\mathrm{MS}$ & $\mathrm{M}$ & $\mathrm{MB}$ & $\mathrm{MB}$ & $\mathrm{B}$ \\
$\mathrm{PB}$ & $\mathrm{M}$ & $\mathrm{MB}$ & $\mathrm{MB}$ & $\mathrm{B}$ & $\mathrm{B}$ \\
\hline
\end{tabular}


where error $e(t)$ is described as $e(t)=V^{*}(t)-V(t)$ and $\lambda$ is non-zero positive design parameters. As far as the guideline for the selection of control gains $\lambda$ is concerned, the gains should be chosen such that the error dynamics at the sliding surface $s=0$ will be forced to decay exponentially to zero.

Consider the control of autopilot system of UAV with dynamics (9) to (13) and figure 3; the speed controller is designed as

$$
\delta_{T}^{*}=u=u_{e q}+u_{s}+u_{a}
$$

where $u_{e q}$ is equivalent control law, $u_{s}$ is switching control law and $u_{a}$ is adaptive control law. The equivalent control law $u_{e q}$ is designed using fuzzy logic based sliding mode control technique. The SMC law can be found using Lyapunov theory and defining the Lyapunov function as $v=s^{2} / 2$. According to Lyapunov theory, if the derivative of $v$ is negative definite, this will ensure that the state trajectory will be driven and attracted toward the sliding surface $s$, and once reached; it will remain sliding on it until the origin is reached asymptotically (Yadav \& Gaur 2014). The time derivative of the Lyapunov function $v$ can be calculated as $\dot{v}=s \dot{s}$. In order to make $\dot{v}$ as negative definite, a fuzzy logic control law is proposed in this paper that removes a lot of mathematical complications. It can be designed with the knowledge of the inputs $s$ and $\dot{s}$ and the output variable $u_{e q}$ of the controller. The proposed structure of FLSMAC is given in figure 5a. The universe of discourse with suitable membership functions of the input and output variable for FLSMAC is described in figure $5 \mathrm{~b}$ and $\mathrm{c}$. The resulting rule base is shown in the table 5 .

The switching control law $u_{s}$ is designed as

$$
u_{s}=-\eta \cdot \operatorname{sgn}(s) \text {, }
$$

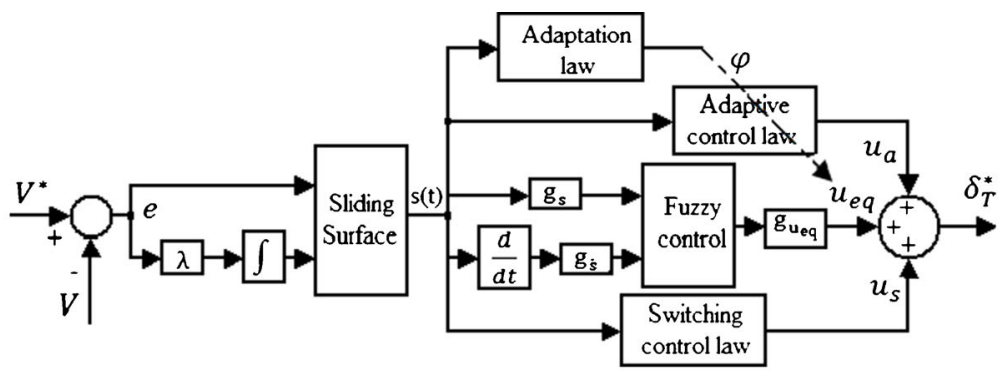

(a)

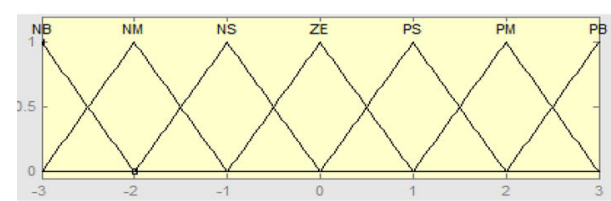

(b)

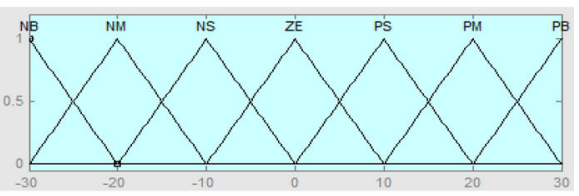

(c)

Figure 5. (a) Structure of proposed FLSMAC. (b) Membership function for input variables $s$ and $\dot{s}$. (c) Membership function for output variable $u_{e q}$. 
Table 5. Rule base matrix of FLSMAC.

\begin{tabular}{llllllll}
\hline$s \backslash \dot{s}$ & NB & NM & NS & ZE & PS & PM & PB \\
\hline NB & NB & NB & NB & NB & NM & NS & ZE \\
NM & NB & NB & NB & NM & NS & ZE & PS \\
NS & NB & NB & NM & NS & ZE & PS & PM \\
ZE & NB & NM & NS & ZE & PS & PM & PB \\
PS & NM & NS & ZE & PS & PM & PB & PB \\
PM & NS & ZE & PS & PM & PB & PB & PB \\
PB & ZE & PS & PM & PB & PB & PB & PB \\
\hline
\end{tabular}

where $\eta$ is hitting control gain and functionsgn is defined as

$$
\operatorname{sgn}(s)=\left\{\begin{array}{lll}
-1 & \text { if } & s<0 \\
+1 & \text { if } & s>0
\end{array}\right\} .
$$

The use of $s g n$ function in the switching control law $u_{s}$ in (30), causes high-frequency chattering due to the discontinuous control action that represents a severe problem when the system state is close to the sliding surface (Yadav \& Gaur 2014). To alleviate the chattering phenomenon, the discontinuous control law term $\operatorname{sgn}(s)$ in (30) is replaced by a $\operatorname{sat}(s)$ function around the switching surface (Sun et al 2011; Zhan \& Zhou 2013; Wai et al 2004; Subudhi \& Ge 2012; Asif et al 2014) the final switching control law is written as

$$
u_{s}=-\eta . s a t(s / \varepsilon) \text {, }
$$

where $\varepsilon>0$ is the width of the boundary, and the function of $s a t(s / \varepsilon)$ is defined as

$$
\operatorname{sat}\left(\frac{s}{\varepsilon}\right)= \begin{cases}1, & \text { for }\left|\left(\frac{s}{\varepsilon}\right)\right| \leq 1 \\ \operatorname{sgn}\left(\frac{s}{\varepsilon}\right), & \text { for }\left|\left(\frac{s}{\varepsilon}\right)\right| \geq 1 .\end{cases}
$$

The adaptive control law $u_{a}$ is designed as

$$
u_{a}=-\frac{s}{\|s\|+\mu} \varphi
$$

with the adaptation law

$$
\dot{\varphi}=\frac{\|s\|^{2}}{\|s\|+\mu},
$$

where $\|$.$\| denotes the standard Euclidean norm and \mu>0$ is chosen such that $\int_{0}^{\infty} \mu d t<\infty$ (Sun et al 2011), then $\dot{s}(t)=0$ due to this the speed tracking error $e(t)$ and $\dot{e}(t)$ will asymptotically converge to zero (Wai et al 2004; Asif et al 2014).

The linguistic variable levels for membership functions used in FL-PD, STF-PID and FLSMAC are assigned as: positive $(\mathrm{P})$, negative $(\mathrm{N})$, positive medium $(\mathrm{PM})$, negative medium (NM), negative big (NB), negative small (NS), zero (ZE), positive small (PS), positive big (PB), small (S), medium small (MS), medium (M), medium big (MB) and big (B). The total control law of the FLSMAC is represented by (28) and could be written in general form as $u_{\text {total }}=u_{e q}+u_{s}+u_{a}$. The equivalent control $u_{e q}$ defines the control action that keeps the 
state trajectory on the sliding surface, $u_{s}$ is the switching control that depends on the sign of the switching surface, and $\eta$ is the hitting control gain that makes derivative of the Lyapunov function i.e., $\dot{v}$ a negative definite (Yadav \& Gaur 2014).

\section{Analysis and interpretation of UAV flights for energy controls}

The energy state of the UAV is defined by its kinetic and potential energy. Kinetic energy is the energy of a moving object and is a function of its speed as given by $E_{k}=W V^{2} / 2 g$. The UAV's potential energy is determined by its altitude above the ground reference as given by $E_{p}=W h$, where ' $h$ ' is the altitude of UAV above the reference, W is the weight of the UAV, $\mathrm{g}$ the gravitational acceleration and $\mathrm{V}$ the airspeed of UAV. The net or total energy is defined as $E=E_{k}+E_{p}$. The only one way in which an UAV can gain total energy is through the energy added by the engine; hence it is managed by throttle position controller of UAV. The net total energy flow into the UAV is a function of the difference between engine thrust and drag as shown in (Amelink et al 2003) i.e., $\dot{E} / V=F_{\text {Thrust }}-F_{\text {Drag }}$. Thus, the throttle does not control speed or altitude like the control strategies discussed here rather controls the UAV total energy balance rate. Since the elevator produces neither drag nor thrust it cannot control the total energy level. It controls the balance between kinetic and potential energy, keeping the net energy constant, hence it is called the energy distribution device. The throttle can be seen as a valve, regulating the total energy flow into the system and the elevator can be seen as a valve distributing the energy flow. The simplified diagram of input output variables of UAV and their cause-effect relations are represented by figure 6 .

In figure 6, the arrows do not represent energy flow or forces. There are three main areas of interest indicated by A, B and C. Section A shows how elevator controls the $\gamma$ and the altitude and section B shows how throttle inputs lead to speed of aerial vehicle after passing through C. Section $\mathrm{C}$ represents the energy relations and forms the link between the elevator control path and throttle control path section A and B respectively. The net or total energy is defined as

$$
E=E_{k}+E_{p}=W h+\frac{1}{2} \frac{W}{g} V^{2}
$$

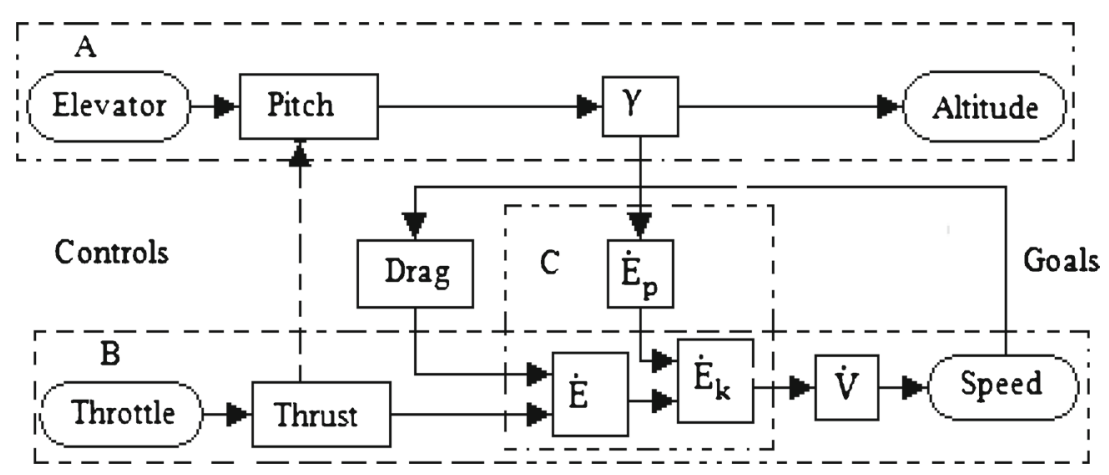

Figure 6. Cause-effect relations between the UAV control variables of autopilot system (Amelink et al 2003). 
and specific energy or energy per unit weight is

$$
\frac{E}{W}=h+\frac{V^{2}}{2 g} .
$$

The specific energy rate $\left(\dot{E}_{S}\right)$ and the specific energy rate per velocity are described in (Akmeliawati \& Mareels 2010) and are given below.

$$
\begin{gathered}
\dot{E}_{s}=\frac{\dot{E}}{W}=\dot{h}+V \frac{\dot{V}}{g}, \\
\frac{\dot{E}_{s}}{V}=\frac{\dot{h}}{V}+\frac{\dot{V}}{g}=\sin \gamma+\frac{\dot{V}}{g} .
\end{gathered}
$$

\section{Simulation results and discussion}

In this section, the results obtained from MATLAB and SIMULINK are presented. In this work, the PI controller is designed as per conventional control technique and fuzzy logic PD, fuzzy PD+I, FLSMAC and STF-PID controller are based on AI-based control. All the controllers as seen in figure 3 are PI controllers. For AI-based control inner loop controllers such as pitch rate, pitch angle and throttle position control are PI controllers but outer loop controllers such as speed and altitude controllers are replaced by fuzzy logic PD, fuzzy PD+I, FLSMAC and STF-PID respectively. All the PI controllers of figure 3 are tuned by Hand-tuning rule through quarter decay ratio of Ziegler-Nichols tuning method. The value of all the designed controllers parameters are given in table 6. The response with PI controllers for pitch rate, pitch angle and throttle position in the inner loop of the line diagram of UAV in figure 3 are shown in figures 7 (a), (b) and (c), respectively.

The air speed and altitude of autopilot system with conventional PI and AI-based controllers like fuzzy logic PD, fuzzy PD+I, FLSMAC as speed controller and STF-PID as altitude controller are represented by figures 8 (a) and (b), respectively. The all phases of UAV such as initial climbs, low altitude flight, climb, cruise, loiter over target zone, descent, initial and final approach and finally landing are also shown in figure 8 (b). The variations in air density during simulation are considered as disturbance here and it is given in figure 8 (c). The IEPI and time domain performance indices of UAV with all designed controllers are given in table 7 . The values of time domain performance index as given in table 7 is determined from enlarged views as seen in figures 8 (a) and (b).

Table 6. Values for controller parameter.

\begin{tabular}{lcclll}
\hline & Conventional PI & & Fuzzy PD & STF-PID & FLSMAC \\
\hline Controller & $\mathrm{K}_{P}$ & $\mathrm{~K}_{I}$ & $\mathrm{~g}_{\mathrm{p}}=0.001$ & $\mathrm{~g}_{\mathrm{e}}=1.2$ & $\lambda=0.1$ \\
Pitch rate & 1.2 & 10 & $\mathrm{~g}_{\mathrm{d}}=0.001$ & $\mathrm{~g}_{\dot{\mathrm{e}}}=0.009$ & $\eta=0.1$ \\
Pitch angle & 3 & 0.01 & $\mathrm{~g}_{\mathrm{u}}=1200$ & $\mathrm{~g}_{\mathrm{p}}=0.01$ & $\mu=100$ \\
Throttle position & 10 & 12 & & $\mathrm{~g}_{\mathrm{i}}=0.01$ & $\mathrm{~g}_{\mathrm{s}}=1 / 400$ \\
Altitude & 0.04 & 0.025 & & $\mathrm{~g}_{\mathrm{d}}=0.01$ & $\mathrm{~g}_{\dot{\mathrm{s}}}=1 / 1500$ \\
Speed & 0.04 & 4.3 & & - & $\mathrm{g}_{\mathrm{u}_{\mathrm{eq}}}=700$ \\
\hline
\end{tabular}




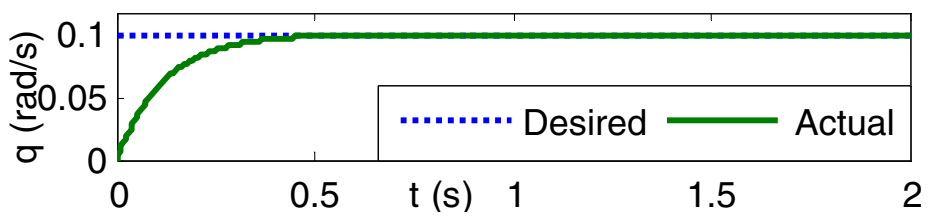

(a)



(b)

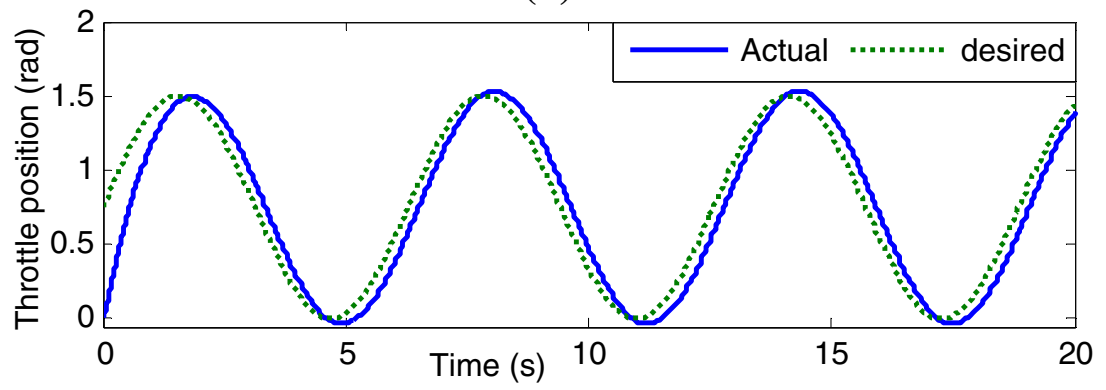

(c)

Figure 7. Response of UAV with PI controller (a) Pitch rate, (b) Pitch angle, (c) Throttle position.

The dynamics of air speed and altitude are dependent on each other as seen in modelling of section 2. But once if autopilot system is stabilized, then they do not affect the performance of each other. This is verifying with the comparisons of results in figures $8(a)$ and (b) and the values of performance index as given in table 7. In figure 8(a), speed is changed at $150 \mathrm{sec}$ and the enlarged view is also shown in the same figure, but it does not affect the response of altitude at the same time. In figure 8 (b), altitudes is changed in steps according to phases of UAV flight, but it does not affect the response of air speed, it is also verified with IAE and ISE values given in table 7, that shows that it is nearly same using STF-PID while speed controllers are different. The enlarged view in figure 8 (b) gives some undesirable and poor transient response; it is due to transition of manual to automatic control. Figure 9 (b) represents the different air speeds such as subsonic, transonic, supersonic and hypersonic speed which are changed in the sequence in every $10 \mathrm{~s}$ and the comparison of PI and AI-based controllers for different airspeed is also presented in same figure.

The PI and AI-based controllers are designed for the UAV in the present work for wide speed range as shown in figure 9. The air speed changes in four steps of $10 \mathrm{~s}$ each that is from $0-100$, $100-400,400-1000$ and $1000-2000 \mathrm{~m} / \mathrm{s}$ and finally $2000 \mathrm{~m} / \mathrm{s}$ to $1500 \mathrm{~m} / \mathrm{s}$ in last $10 \mathrm{~s}$ as shown in figure 9. From figure 8 (a), figure 9 and table 7, it is concluded that the response of air speed with FLSMAC is far better than the response of conventional PI and other designed AI-based controllers. It gives least OS, RT, ST, steady state error $\left(\mathrm{E}_{s s}\right)$, IAE and ISE as compared to other designed controllers. The response of altitude with STF-PID is far better than the response of PI 


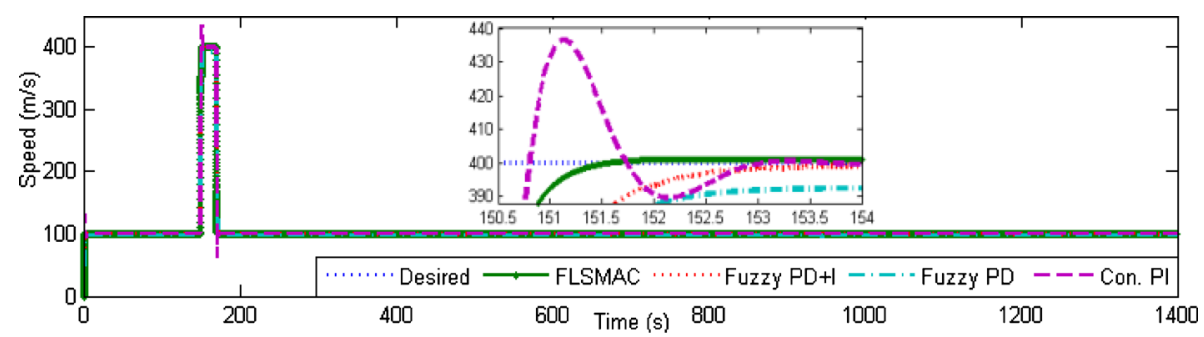

(a)

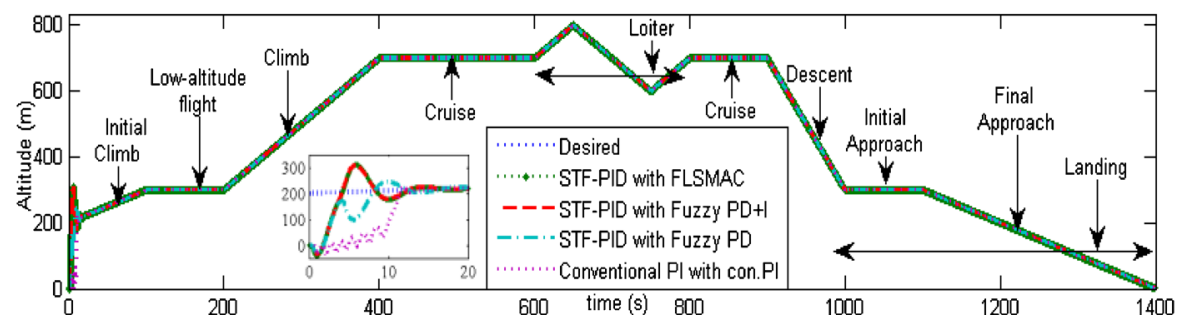

(b)

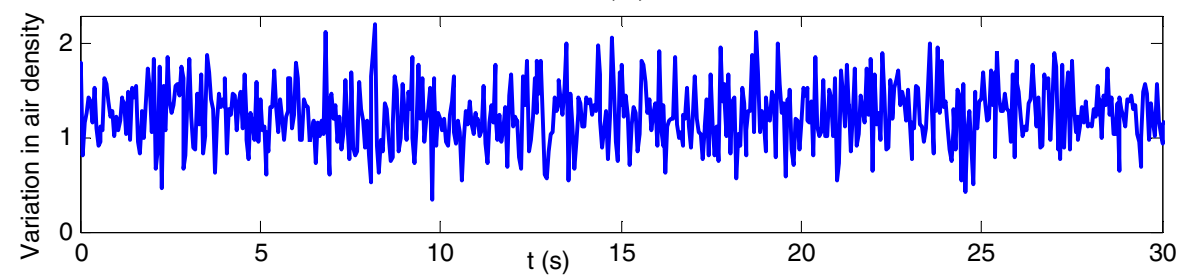

(c)

Figure 8. (a) UAV speed control with conventional PI and AI based control. (b) UAV Altitude control with conventional PI and AI-based control. (c) Variation in air density as disturbance.

controller as clearly shown with values of ISE and IAE as given in table 7. According to proper balance in time domain and IEPI, autopilot system for UAV with FLSMAC as speed controller and STF-PID as altitude gives best control performance.

In order to see the effect of air density disturbance rejection and mass uncertainty of autopilot system using the proposed FLSMAC technique, the constant sliding surface parameter $\lambda$ is modified to a time varying parameter $\lambda(t)$ and estimated online according to adaptation rule

Table 7. Performance index of autopilot system with different combination of controllers.

\begin{tabular}{|c|c|c|c|c|c|c|c|c|c|c|c|}
\hline \multicolumn{7}{|c|}{ Speed } & \multicolumn{5}{|c|}{ Altitude } \\
\hline$\overline{\text { Perf. index } \rightarrow}$ & & IEPI & & Time & domain & & & IEPI & & Time & domain \\
\hline Controller & IAE & ISE & RT (s) & $\mathrm{ST}(\mathrm{s})$ & OS $(\%)$ & $\mathrm{E}_{s s}(\%)$ & Controller & IAE & ISE & ST (s) & OS $(\%)$ \\
\hline PI & 386.9 & $6.3 * 10^{4}$ & 0.71 & 1.5 & 9.5 & 0.01 & PI & 2035 & $3.8^{*} 10^{5}$ & 18.2 & 12.8 \\
\hline Fuzzy PD & 3099 & $5.33 * 10^{4}$ & 1.32 & 1.91 & 0.0 & 1.8 & STF-PID & 1074 & $1.42 * 10^{5}$ & 15.7 & 23.6 \\
\hline Fuzzy PD+I & 604.2 & $4.79 * 10^{4}$ & 1.1 & 1.54 & 0.0 & 0.02 & STF-PID & 1032 & $1.38 * 10^{5}$ & 12.4 & 54.3 \\
\hline FLSMAC & 212.5 & $2.63 * 10^{4}$ & 0.76 & 0.87 & 0.0 & 0.012 & STF-PID & 1046 & $1.41 * 10^{5}$ & 11.8 & 52.72 \\
\hline
\end{tabular}




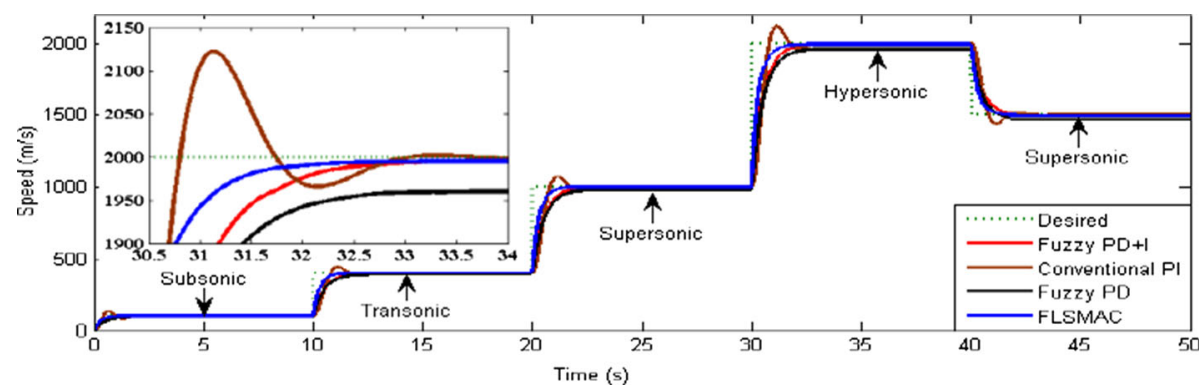

Figure 9. Various UAV airspeeds with PI and AI-based controllers.

$\left.\dot{\lambda}(t)=-\vartheta . s(t) . \int^{(} V^{*}(t)-V(t)\right) d t$, where $\vartheta$ is a constant. To test disturbance, i.e., air density rejection and effect of mass uncertainty on speed and altitude performance of UAV using FLSMAC with constant $\lambda$ and then with time varying sliding surface parameter $\lambda(t), a+100$ air density at $\mathrm{t}=60 \mathrm{~s}$ and $+10,000 \mathrm{~kg}$ mass at $\mathrm{t}=80 \mathrm{~s},-12,500 \mathrm{~kg}$ mass at $\mathrm{t}=81 \mathrm{~s}$ and $0 \mathrm{~kg}$ mass at $\mathrm{t}=82 \mathrm{~s}$ is applied and the effect on response of speed and altitude with constant $\lambda$ and then with time varying $\lambda(t)$ of FLSMAC is represented in figures 10 (a) and (b). In figures 10 (a) and (b) both disturbances due to air density as well as mass uncertainty is taken into consideration. From figure 10 (a) it is clearly seen that the effect of air density and mass uncertainty on air speed with FLSMAC using constant $\lambda$ is more as compared to FLSMAC with time varying $\lambda(t)$.

The FLSMAC with $\lambda(t)$ gives small $5.7 \%$ OS and nearly $0.9 \mathrm{~s}$ settling time. These disturbances are not affecting the altitude performance because STF-PID controller is used as altitude controller as seen from figure 10(b). Hence, the autopilot system for UAV using FLSMAC with time varying $\lambda$ gives robust and adaptive performance.

Due to the significant steady state error in air speed using AI-based control technique, the kinetic energy may not be accurately balanced and this is reflected as minor deviation in specific

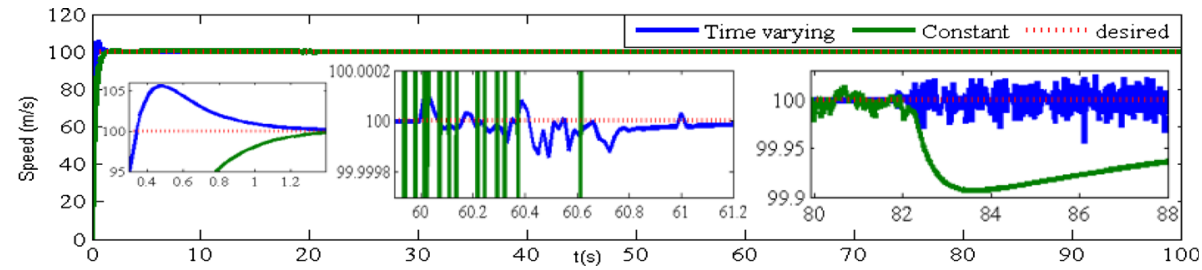

(a)

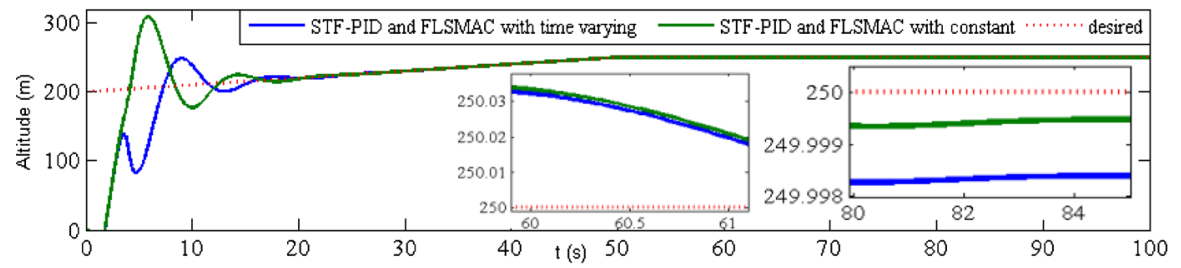

(b)

Figure 10. Response with constant and time varying $\lambda$ of FLSMAC (a) speed, (b) altitude. 


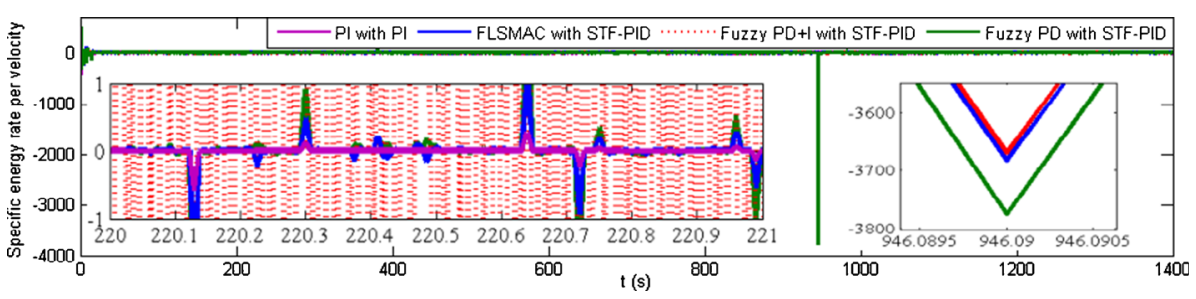

Figure 11. Response of specific energy rate per velocity.

energy rate per velocity for short duration in UAV as seen in figure 11 and that may be neglected as it is minor deviation in energy level and within the limits.

As seen from figure 11, specific energy rate per velocity is finally zero for a specified flight path when autopilot system is activated between the interval of $10 \mathrm{~s}$ to the $1400 \mathrm{~s}$, with constant air speed of $100 \mathrm{~m} / \mathrm{s}$ throughout the path. During the transients, the controller regulates the kinetic energy well and the potential energy is expected to rise. The plot is drawn using (37) ' $\sin \gamma+\dot{V} / g$ ' for specific energy rate per velocity as shown in figure 11 . As can be seen, the total energy is balanced at fast rate after every transient hence the energy control efficiency is achieved. The objective of the analysis is to verify the robustness of the proposed closedloop autopilot system against various flight conditions and to investigate the conformity of the closed-loop system responses. In the present work, the main concern is the effect on the aircraft energy management by controlling the aircraft speed and altitude and the analysis of transient and steady-state response for various control strategies on closed-loop system during the transient period, to investigate the rise time, the settling time and the air speed responses. According to proper balance in time domain, IEPI and energy control of autopilot system for UAV with FLSMAC as speed controller and STF-PID as altitude give the best control performance.

\section{Conclusion}

In this paper, the design of autopilot system for longitudinal motion of UAV is presented. The AI-based STF-PID controller as altitude control and FLSMAC as speed control are designed to improve the UAV performance and also to reduce the controller tuning time effectively, hence replacing conventional PI controller where a large time is required for tuning. The conventional PI and AI-based controllers are applied to control the speed and altitude by mixing appropriate amount of air mass to the fuel mass through throttle valve of autopilot system for nonlinear UAV. The results show that AI-based controller give better performance results in comparison to conventional PI controllers considered for inner and outer loops in UAV control. Since the maximum overshoot and settling time in achieving the desired speed being the least with AIbased controller, hence the current and torque of the motor to control the same will also be optimized with the desired throttle position of ETCS through DC servo motor which ensures better fuel economy and efficiency. AI-based control technique may also be applied to other similar nonlinear systems for performance optimization. Since the air mass flow to the fuel mass is dependent on throttle position and the ratio of the same decides the energy efficiency, fuel economy and pollution, it may be concluded that UAV along with AI-based control of ETCS is effectively controlled for variable speed operation of the same. 


\section{References}

Akmeliawati R and Mareels I M Y 2010 Nonlinear energy based control method for aircraft automatic landing systems. IEEE Transactions on Control Systems Technology, 18(4): 871-884

Amelink M H J, Renévan Paassen M M, Mulder M and Flach J M 2003 Applying the abstraction hierarchy to the aircraft manual control task, Proceedings of the 12th International Symposium on Aviation Psychology, Dayton, USA

Asif M, Khan M J and Cai N 2014 Adaptive sliding mode dynamic controller with integrator in the loop for nonholonomic wheeled mobile robot trajectory tracking. Int. J. Control, 87(3): 964-975

Bateman F O, Noura H and Ouladsine M 2011 Fault diagnosis and fault-tolerant control strategy for the aerosonde UAV. IEEE Transactions on Aerospace and Electronic Systems, 47(3): 2119-2137

Chao H Y, Cao Y C and Chen Y Q 2010 Autopilots for small unmanned aerial vehicles: a survey. International Journal of Control, Automation, and Systems, 8(1): 36-44

Ducard G J J 2009 Fault-tolerant flight control and guidance system: practical methods for small unmanned aerial vehicles. London: Springer-Verlog London Limited

Goerzen C, Kong Z and Mettler B 2010 A survey of motion planning algorithms from the perspective of autonomous UAV guidance. Journal of Intelligent Robot System, 57: 65-100

Gu Y, Seanor B, Campa G, Napolitano M R, Rowe L, Gururajan S and Wan S 2006 Design and flight testing evaluation of formation control laws. IEEE Transactions on Control Systems Technology, 14(6): $1105-1112$

Han Y, Oh S, Choi B, Kwak D, Kim H J and Kim Y 2012 Fault detection and identification of aircraft control surface using adaptive observer and input bias estimator. IET Control Theory Appl. 6(7): 13671387

Hull D G 2009 Fundamentals of airplane flight mechanics. London: Springer Verlog London Limited

Kamalasadan S and Ghandakly A A 2011 A neural network parallel adaptive controller for fighter aircraft pitch-rate tracking. IEEE Transactions on Instrumentation and Measurement, 60(1): 258-267

Keviczky T and Balas G J 2006 Receding horizon control of an F-16 aircraft: A comparative study. Control Engineering Practice, 14: 1023-1033

Kontogiannis S G and Ekaterinaris J A 2013 Design, performance evaluation and optimization of a UAV. Aerospace Science and Technology, 29: 339-350

Kurnaz S, Cetin O and Kaynak O 2009 Fuzzy logic based approach to design of flight control and navigation tasks for autonomous unmanned aerial vehicles. Journal of Intelligent Robot System, 54: 229-244

Liu C, Aree O M and Chen W H 2013 Path-following control for small fixed-wing unmanned aerial vehicles under wind disturbances. Int. J. Robust and Nonlinear Control, 23: 1682-1698

Liu Y, Tang X, Tao G and Joshi S M 2008 Adaptive compensation of aircraft actuation failures using an engine differential model. IEEE Transactions on Control Systems Technology, 16(3): 971-982

Li Y and Xu Q 2010 Adaptive sliding mode control with perturbation estimation and pid sliding surface for motion tracking of a piezo-driven micromanipulator. IEEE Transactions on Control Systems Technology, 18(4): 798-810

Manathara J G and Debasish G 2011 Reactive collision avoidance of multiple realistic UAVs. Aircraft Engineering and Aerospace Technology: An International Journal, 83(6): 388-396

Orsag M, Bogdan S, Haus T, Bunic M and Krnjak A 2011 Modeling, simulation and control of a Spin Copter, Proceedings IEEE International Conference on Robotics and Automation. Shanghai, China: Shanghai International Conference Center

Osborne R W, Shalom Y B, Willett P and Baker G 2011 Design of an adaptive passive collision warning system for UAVs. IEEE Transactions on Aerospace and Electronic Systems, 47(3): 2169-2189

Shiau J K and Ma D-M 2009 An autopilot design for the longitudinal dynamics of a low speed experimental UAV using two time scale cascade decomposition. Transactions of the Canadian Society for Mechanical Engineering, 33(3): 501-521

Shin Y C and Xu C 2009 Intelligent systems; modeling, optimization, and control. New York: CRC Press, Taylor and Francis Group 
Subudhi B and Ge S S 2012 Sliding-mode-observer-based adaptive slip ratio control for electric and hybrid vehicles. IEEE Transactions on Intelligent Transportation Systems, 13(4): 1617-1626

Sun T, Pei H, Pan Y, Zhou H and Zhang C 2011 Neural network-based sliding mode adaptive control for robot manipulators. Neurocomputing, 74: 2377-2384

Stevens B L and Lewis F L 2003 Aircraft Control and Simulation, John Wiley \& Sons, Inc., 2nd edition Hoboken, New Jersey

Vasak M, Baotic M, Petrovic I and Peric N 2007 Hybrid theory-based time optimal control of an electronic throttle. IEEE Transactions on Industrial Electronics, 54(3): 1483-1494

Wai R J, Lin C M and Hsu C F 2004 Adaptive fuzzy sliding-mode control for electrical servo drive. Fuzzy Sets and Systems, 143: 295-310

Wang Q and Stengel R F 2000 Robust nonlinear control of a hypersonic aircraft. Journal of Guidance, Control, and Dynamics, 23(4): 577-585

Wang Q and Stengel R F 2005 Robust nonlinear flight control of a high-performance aircraft. IEEE Transactions on Control Systems Technology, 13(1): 15-26

Yadav A K and Gaur P 2013 Comparative analysis of modern control and AI-based control for maintaining constant ambient temperature. World Review of Science, Technology and Sustainable Development, 10(1-3): 56-77

Yadav A K and Gaur P 2014 Robust adaptive speed control of uncertain hybrid electric vehicle using electronic throttle control with varying road grade. An International Journal of Nonlinear Dynamics and Chaos in Engineering Systems, 76(1): 305-321

Yadav A K, Gaur P, Jha S K, Gupta J R P and Mittal A P 2011a Optimal speed control of hybrid electric vehicles. Journal of Power Electronics, 11(4): 393-400

Yadav A K, Gaur P, Mittal A P and Anzar M 2011b Comparative analysis of various control techniques for inverted pendulum, Proc. of IICPE-2010, New Delhi

Zhan L and Zhou K 2013 Adaptive fuzzy sliding mode control for a robotic aircraft flexible tooling system. Int. J. Adv. Manuf. Technology, 69: 1469-1481

Zulfatman and Rahmat M F 2009 Application of self-tuning fuzzy PID controller on industrial hydraulic actuator using system identification approach. International Journal on Smart Sensing and Intelligent Systems, 2(2): 246-261 